Canadian Oncology

Nursing Journal

Revue canadienne

de soins infirmiers

en oncologie

Volume 31, Issue 1 • Winter 2021

elSSN: 2368-8076 


\title{
Écrire entre les lignes : analyse secondaire des commentaires non sollicités de survivants du cancer sur la peur d'une récidive
}

\author{
par Jacqueline Galica, Stephanie Saunders, Kristen Haase et Christine Maheu
}

\section{RÉSUMÉ}

Contexte : La peur de récidive du cancer (PRC) survient couramment chez les survivants du cancer après leur traitement. La présente analyse secondaire explore les commentaires non sollicités de survivants du cancer dans le cadre d'un sondage sur la PRC.

Méthodologie: La présente étude a fait appel à la méthode descriptive interprétative et aux analyses statistiques pour explorer les commentaires non sollicités et déterminer les caractéristiques respectives des survivants ayant étoffé leurs réponses et de ceux ne l'ayant pas fait.

Résultats : Trois thèmes sont ressortis de l'analyse : description de l'expérience post-traitement, approfondissement ou contextualisation des réponses sur la $P R C$, et expression de la nécessité d'améliorer les soins oncologiques. De façon générale, chez les personnes ayant fourni des commentaires non sollicités, la PRC était moindre. La plupart du temps, les commentaires visaient à contextualiser certaines réponses ou à signaler la non-pertinence de certaines questions.

Conclusion : L'étude met de l'avant, d'une part, diverses raisons expliquant les commentaires non sollicités dans le cadre d'un sondage et, d'autre part, la valeur potentielle de la prise de parole des survivants du cancer. Les résultats témoignent de l'efficacité d'une approche méthodologique mixte qui intégrerait au sondage un espace où les répondants peuvent s'exprimer librement.

Mots-clés : survivants du cancer; peur; commentaires; sondages et questionnaires; étude qualitative

T a peur de récidive du cancer (PRC), qui se rapporte à la crainte ـou à l'inquiétude qu'un cancer revienne ou évolue (Lebel,

\section{AUTEURES}

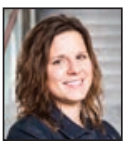

Jacqueline Galica, inf. aut., Ph.D., CSIO(C); professeure adjointe, École des sciences infirmières, Université Queen's; membre à part entière, Division des soins oncologiques et de l'épidémiologie, Institut de recherche sur le cancer de l'Université Queen's; jacqueline.galica@queensu.ca

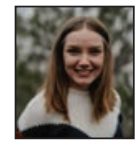

Stephanie Saunders, étudiante au B.Sc.inf.; École des sciences infirmières, Université Queen's; sms21@queensu.ca

Kristen Haase, inf. aut., Ph.D.; professeure adjointe, École des sciences infirmières, Université de la Colombie-Britannique; kristenrhaase@ubc.ca

Christine Maheu, inf. aut., Ph.D.; professeure adjointe, École de sciences infirmières Ingram, Université McGill; christine.maheu@ mcgill.ca

DOI:10.5737/2368807631192101
Ozakinci, et al., 2016), affecte jusqu'à $72 \%$ des survivants du cancer (Costa, Dieng, Cust, Butow et Kasparian, 2016). Une certaine PRC est une réaction normale au cancer (Lebel, Ozakinci, et al., 2016), mais trop grande, la PRC nuit gravement au bienêtre psychologique. En effet, une PRC élevée serait associée à une hausse de détresse (Hall et al., 2019; Mehnert, Koch, Sundermann et Dinkel, 2013), à de l'anxiété (Eyrenci et Sertel Berk, 2018; Simard et Savard, 2009) et à une baisse de qualité de vie (Lebel, Simard et al., 2016; Liu et al., 2017; Simard et Savard, 2009). Elle serait aussi corrélée à un usage accru des ressources en santé (Lebel, Tomei, Feldstain, Beattie et McCallum, 2013) et à une augmentation des comportements mésadaptés (Hall et al., 2019), ce qui laisse croire qu'elle nuit à la capacité des survivants à composer avec leur cancer. Considérés dans leur ensemble, ces résultats soulignent l'importance d'explorer la PRC des survivants du cancer ayant terminé leur traitement.

En 2015, l'auteure principale (J. G.) a mené une enquête transversale à mode mixte, conçue pour examiner les variables prédictives et médiatrices de la PRC (Galica, Maheu, Brennenstuhl, Townsley et Metcalfe, 2019). Au total, 1002 survivants du cancer (échantillon hétérogène) ont participé aux sondages, lesquels intégraient des mesures valides et fiables (voir tableau 1). Le recrutement s'est fait dans une clinique pour les survivants du cancer où les patients étaient suivis pendant cinq ans avant d'être redirigés vers leur fournisseur de soins primaires. Les données cliniques (sur le diagnostic et le traitement, par exemple) ont été extraites des dossiers cliniques par l'auteure principale. À la lecture des sondages, l'équipe de recherche a remarqué que certains participants avaient ajouté des commentaires de leur propre chef dans les marges ou entre les questions, ou encore avaient joint des lettres manuscrites ou tapées à l'ordinateur. Les commentaires prenaient différentes formes : phrases courtes, paragraphes couvrant une à plusieurs marges, lettres rédigées sur d'autres feuilles. Léquipe de recherche s'est interrogée sur les motifs de ces ajouts et, étant donné que ces derniers ne faisaient pas l'objet de l'analyse des sondages, ils ont été consignés dans une base de données pour la présente analyse secondaire.

Peu d'études traitent des commentaires non sollicités reçus dans le cadre d'une recherche par sondage (Clayton, Rogers et Stuifbergen, 1999; Maliski et Litwin, 2007; Marshall, Linden, Ng, et Vodermaier, 2019; Warms, Marshall, Hoffman et Tyler, 2005), des commentaires susceptibles d'apporter un éclairage supplémentaire sur l'expérience des participants (Clayton et al., 1999; Maliski et Litwin, 2007; Warms et al., 2005). Par « commentaires non sollicités ", on entend les témoignages écrits qui sortent du cadre d'analyse de l'étude (Frid, Ohlen et Bergbom, 2000). Ainsi, les répondants émettent parfois des commentaires 
Tableau 1. Sondages réalisés par les participants dans l'étude principale

\begin{tabular}{|l|l|c|}
\hline Variable & Mesure & $\begin{array}{c}\text { Nbre (\%) de } \\
\text { commentaires }(\mathbf{n}=124)^{2}\end{array}$ \\
\hline Situation sociodémographique & $\begin{array}{l}\text { Fiche de données démographiques (créée par l'équipe de recherche pour } \\
\text { l'étude principale) }\end{array}$ & $12(9,7 \%)$ \\
\hline Peur de la récidive du cancer & Inventaire de la peur de récidive du cancer (IPRC) (Simard et Savard, 2009) & $58(46,8 \%)$ \\
\hline Estime de soi & Échelle d'estime de soi de Rosenberg (Rosenberg, 1965) & $2(1,6 \%)$ \\
\hline Optimisme-pessimisme & Revised Life Orientation Test (LOT-R) (Scheier et Carver, 1985) & 0 (0,0\%) \\
\hline Représentation de la maladie & Revised Illness Perception Questionnaire (IPQ-R) (Moss-Morris et al., 2002) & $24(19,4 \%)$ \\
\hline Personnalité & Big Five Inventory à 10 items (BFI-10) (Rammstedt et John, 2007) & $2(1,6 \%)$ \\
\hline $\begin{array}{l}\text { Styles et stratégies d'adaptation } \\
\text { 2Au total, 18 commentaires (14,5 \%) ont été rédigés sur des documents à part (lettres ou papillons adhésifs), et joints aux sondages ensuite } \\
\text { rendus. }\end{array}$ & $8(6,5 \%)$ \\
\hline
\end{tabular}

spontanés dans les entretiens (Warms et al., 2005), ou sont amenés à expliquer, à étoffer ou à contextualiser des réponses (Maliski et Litwin, 2007) pour pallier certaines lacunes des sondages (Marshall et al., 2019). Précieuse source de données, les commentaires écrits font état des témoignages personnels des survivants (O'Brien et Clark, 2010), qui échappent peut-être aux autres méthodes de recherche.

Étant donné que les commentaires non sollicités peuvent éclairer les expériences de ceux qui les ont formulés (Maliski et Litwin, 2007) et aider les patients cancéreux et les survivants à composer avec la maladie (Carlick et Biley, 2004), l'équipe de recherche s'est penchée sur les commentaires non sollicités de survivants du cancer ayant participé à une enquête sur la PRC. L'analyse a été faite à la lumière de trois objectifs : 1) Examiner ce qui motive ces commentaires non sollicités fournis dans le cadre d'une série de sondages; 2) Déterminer les caractéristiques des participants ayant fourni des commentaires non sollicités; 3) Évaluer si la situation démographique et clinique ainsi que le degré de PRC diffèrent entre les participants ayant fourni des commentaires non sollicités et ceux n'en ayant pas fourni.

\section{MÉTHODES}

Aux fins de cette analyse secondaire, de multiples méthodes de recherche (Hunter et Brewer, 2016) ont été utilisées pour répondre aux objectifs fixés. D'ailleurs, tous les participants ont donné leur consentement éclairé dans le cadre de la première étude (Galica et al., 2019), et le comité déthique de la recherche en sciences de la santé de l'Université Queen's a autorisé la présente analyse secondaire (\#6026530).

\section{Dimension qualitative}

Pour ce qui est de la dimension qualitative (objectif 1), une méthode descriptive interprétative (Thorne, 2013, 2016) a servi à analyser les commentaires non sollicités reçus (tous sondages confondus). La description interprétative invite les chercheurs à transcender les méthodes traditionnelles pour intégrer leurs connaissances disciplinaires à la démarche de recherche. Grâce à cette approche qualitative, les données s'ancrent dans un contexte clinique pertinent qui contribue à l'orientation et à la compréhension de la réalité infirmière (Thorne, 2016). À l'aide de Microsoft Word et Microsoft Excel, deux chercheuses (J. G., S. S.) ont indépendamment

\begin{tabular}{|c|c|}
\hline Principe & Stratégies adoptées \\
\hline $\begin{array}{l}\text { Intégrité } \\
\text { épistémologique }\end{array}$ & $\begin{array}{l}\text { - Conception adaptée aux objectifs de } \\
\text { recherche }\end{array}$ \\
\hline Crédibilité & $\begin{array}{l}\text { - Codage indépendant et définition des thèmes } \\
\text { par plus de deux membres de l'équipe de } \\
\text { recherche } \\
\text { - Représentativité des codes et des thèmes, } \\
\text { qui reflètent la diversité des perspectives des } \\
\text { participants }\end{array}$ \\
\hline $\begin{array}{l}\text { Logique } \\
\text { analytique }\end{array}$ & - Piste de vérification des décisions clés \\
\hline $\begin{array}{l}\text { Aptitude à } \\
\text { interpréter }\end{array}$ & $\begin{array}{l}\text { - Utilisation de citations à titre d'exemples } \\
\text { - Représentativité des exemples, qui reflètent } \\
\text { la diversité des participants }\end{array}$ \\
\hline
\end{tabular}

codifié ligne par ligne les commentaires non sollicités des participants. À mesure que les analyses s'approfondissaient, ces deux mêmes chercheuses répartissaient les codes dans des sous-catégories, qu'elles réorganisaient régulièrement en vue de mettre au point les thèmes du cadre de travail initial. Les désaccords entourant le classement des codes et le choix des thèmes ont été réglés grâce aux conseils d'une troisième chercheuse (C. M.). Le cadre a évolué tout au long de l'analyse, en consultation avec l'équipe de rédaction (K. H., C. M.).

Pour assurer la rigueur de l'analyse qualitative, l'équipe de recherche a suivi les principes de fiabilité de Thorne (2016) tout au long du processus : intégrité épistémologique, crédibilité, logique analytique et aptitude à interpréter (Thorne, 2016). Les considérations et les stratégies de Thorne (2016) ayant guidé la démarche figurent dans le tableau 2.

\section{Dimension quantitative}

La dimension quantitative s'est traduite par un examen statistique des données de l'étude principale se rapportant aux 47 participants ayant fourni des commentaires non sollicités, tous sondages confondus (objectif 2). La version 25 du logiciel SPSS a été utilisée pour examiner, d'un point de vue descriptif, les variables démographiques et cliniques (voir tableaux 3 et 4) et le degré de PRC (voir ci-dessous) des 


\begin{tabular}{|c|c|c|c|c|c|c|}
\hline Variable & \multicolumn{2}{|c|}{$\begin{array}{l}\text { Personnes ayant laissé des } \\
\text { commentaires }(n=47)\end{array}$} & \multicolumn{2}{|c|}{$\begin{array}{l}\text { Personnes n'ayant pas laissé de } \\
\text { commentaires }(n=955)\end{array}$} & Test statistique $^{\mathrm{a}}$ & $p$ \\
\hline \multirow[t]{2}{*}{ Âge } & 66,0 & 12,1 & 60,9 & 11,9 & $t=-2,90$ & $<.01$ \\
\hline & $\mathbf{N}$ & $\%$ & $\mathbf{N}$ & $\%$ & & \\
\hline Femme & 39 & 83,0 & 813 & 85,3 & & \\
\hline Homme & 8 & 17,0 & 140 & 14,7 & & \\
\hline État matrimonial & & & & & 1,62 & .20 \\
\hline $\begin{array}{l}\text { Marié(e) ou conjoint(e) de } \\
\text { fait }\end{array}$ & 28 & 59,6 & 653 & 68,4 & & \\
\hline Oui & 34 & 72,3 & 701 & 73,4 & & \\
\hline Non & 13 & 27,7 & 254 & 26,6 & & \\
\hline Enfants à charge & & & & & 0,76 & .38 \\
\hline Oui & 5 & 10,6 & 146 & 15,3 & & \\
\hline Non & 42 & 89,4 & 809 & 84,7 & & \\
\hline Niveau d'éducationb & & & & & 8,90 & $<.01$ \\
\hline $\begin{array}{l}\text { Diplôme d'études } \\
\text { postsecondaires (études } \\
\text { universitaires parfois } \\
\text { entreprises, mais non } \\
\text { terminées) }\end{array}$ & 11 & 23,4 & 435 & 45,5 & & \\
\hline En emploi & 19 & 40,4 & 440 & 46,2 & & \\
\hline Sans emploi & 28 & 59,6 & 513 & 53,8 & & \\
\hline Ascendance & & & & & 0,88 & .35 \\
\hline Blanc/Caucasien & 39 & 83,0 & 734 & 77,1 & & \\
\hline Autres origines ethniques & 8 & 17,0 & 218 & 22,9 & & \\
\hline Originaire du Canada & & & & & 3,90 & .04 \\
\hline Oui & 33 & 70,2 & 529 & 55,6 & & \\
\hline Non & 14 & 29,8 & 423 & 44,4 & & \\
\hline Emplacement géographique & & & & & $\mathrm{FE}=0,11$ & .05 \\
\hline Milieu urbain & 47 & 100 & 897 & 93,9 & & \\
\hline Milieu rural & 0 & 0 & 58 & 6,1 & & \\
\hline $\begin{array}{l}\text { Note : FE = méthode exacte de } \\
p=\text { niveau de signification (test } \\
\text { fréquences n'égale pas le total } \\
\text { a } \times 2=\text { Test du khi-carré de Pears } \\
\text { d'études postsecondaires (étud } \\
\text { études au secondaire ou dans ur } \\
\text { ou d'un niveau d'études supérie }\end{array}$ & $\begin{array}{l}\text { er (utilise } \\
\text { éral); } t= \\
\text { lonnes. } \\
\text { moins d' } \\
\text { iversitair } \\
\text { ole comn }\end{array}$ & $\begin{array}{l}\text { nné le no } \\
\text { pendant. } \\
\text { contraire } \\
\text { ntreprise } \\
\text { /techniqu } \\
\text { sonnes à }\end{array}$ & $\begin{array}{l}\text { d'élément } \\
\text { aison de dor } \\
\text { niveau d'é } \\
\text { is non term } \\
\text { commencé } \\
\text { terminé leu }\end{array}$ & $\begin{array}{l}\text { certaines cel } \\
\text { quantes, il se } \\
\text { été réparti e } \\
\text { ui regroupe } \\
\text { s universitai } \\
\text { éat ou des é }\end{array}$ & $\begin{array}{l}\text { ules. Probabilité ex } \\
\text { peut que le total c } \\
\text { deux catégories: } \\
\text { es personnes ayant } \\
\text { es, et « Diplôme de } \\
\text { udes postérieures }\end{array}$ & $\begin{array}{l}\text { consignée); } \\
\text { taines } \\
\text { olôme } \\
\text { niné leurs } \\
\text { mier cycle } \\
\text { cccalauréat. }\end{array}$ \\
\hline
\end{tabular}




\begin{tabular}{|c|c|c|c|c|c|c|}
\hline \multirow[t]{2}{*}{ Variable } & \multicolumn{2}{|c|}{$\begin{array}{l}\text { Personnes ayant laissé des } \\
\text { commentaires }(n=47)\end{array}$} & \multicolumn{2}{|c|}{$\begin{array}{l}\text { Personnes n'ayant pas laissé } \\
\text { de commentaires }(n=955)\end{array}$} & \multirow[t]{2}{*}{$\begin{array}{c}\text { Test } \\
\text { statistique }^{a}\end{array}$} & \multirow[t]{2}{*}{$p$} \\
\hline & $\overline{\mathbf{x}}$ & SD & $\overline{\mathbf{x}}$ & SD & & \\
\hline Temps écoulé depuis le diagnostic (années) & 10,77 & 6,86 & 8,99 & 5,02 & $t=-1,75$ & .09 \\
\hline \multirow[t]{2}{*}{ Nombre de comorbidités } & 1,26 & 1,36 & 0,79 & 0,94 & $t=-2,31$ & .03 \\
\hline & $\mathbf{N}$ & $\%$ & $N$ & $\%$ & & \\
\hline Type de cancer & & & & & 0,45 & .50 \\
\hline Cancer du sein & 29 & 61,7 & 632 & 66,5 & & \\
\hline Autres cancers & 18 & 38,3 & 319 & 33,5 & & \\
\hline Stade de l'AJCC & & & & & 2,67 & .10 \\
\hline $0-1$ & 25 & 53,2 & 393 & 41,2 & & \\
\hline 2-4 (et stade inconnu) & 22 & 46,8 & 562 & 58,8 & & \\
\hline Chimiothérapie & & & & & 2,36 & .12 \\
\hline Oui & 21 & 44,7 & 529 & 56,1 & & \\
\hline Non & 26 & 55,3 & 414 & 43,9 & & \\
\hline Radiothérapie & & & & & 0,04 & .84 \\
\hline Oui & 31 & 66,0 & 609 & 64,5 & & \\
\hline Non & 16 & 34,0 & 335 & 35,5 & & \\
\hline Autre traitement & & & & & 1,40 & .24 \\
\hline Oui & 26 & 55,3 & 602 & 63,8 & & \\
\hline Non & 21 & 44,7 & 341 & 36,2 & & \\
\hline Tout traitement du cancer & & & & & 0,46 & .50 \\
\hline Oui & 41 & 87,2 & 852 & 90,3 & & \\
\hline Non & 6 & 12,8 & 92 & 9,7 & & \\
\hline Autre cancer primitif/récidive/ métastase & & & & & 12,84 & $<.01$ \\
\hline Oui & 21 & 44,7 & 211 & 22,1 & & \\
\hline Non & 26 & 55,3 & 744 & 77,9 & & \\
\hline $\begin{array}{l}\text { Lien avec la clinique pour les survivants du } \\
\text { cancer }\end{array}$ & & & & & 3,29 & .07 \\
\hline Suivi actif & 29 & 61,7 & 704 & 73,7 & & \\
\hline En congé & 18 & 38,3 & 251 & 26,3 & & \\
\hline Connaît quelqu'un dont le cancer a récidivé & & & & & 2,60 & .11 \\
\hline Oui & 28 & 59,6 & 454 & 47,5 & & \\
\hline Non/Ne sait pas & 19 & 40,4 & 501 & 52,5 & & \\
\hline $\begin{array}{l}\text { Connaît quelqu'un pour qui la récidive a } \\
\text { influencé la PRC }\end{array}$ & & & & & 1,66 & .20 \\
\hline Oui & 8 & 17,0 & 242 & 25,3 & & \\
\hline Non/Ne sait pas & 39 & 83,0 & 713 & 74,7 & & \\
\hline $\begin{array}{l}\text { Note }: p=\text { niveau de signification (test bilaté } \\
\text { certaines fréquences n'égale pas le total des } \\
a \times 2=\text { Test du khi-carré de Pearson, à moins }\end{array}$ & $\begin{array}{l}; t=\text { test }-\mathrm{t} \\
\text { onnes. } \\
\text { dication co }\end{array}$ & ndant. En r & son de don & רquantes, il & e peut que le $t$ & \\
\hline
\end{tabular}


participants ayant formulé des commentaires non sollicités. Ensuite, des tests t pour échantillons indépendants et des tests du khi-carré ont servi à analyser en quoi se distinguent ces variables et le degré de PRC quand on compare les participants ayant fourni des commentaires non sollicités dans le cadre de l'étude principale et ceux ne l'ayant pas fait (objectif 3).

\section{Inventaire de la peur de la récidive du cancer}

Dans le cadre de l'étude principale (Galica et al., 2019), l'Inventaire de la peur de la récidive du cancer (IPRC) (Simard et Savard, 2009) a été utilisé pour évaluer la PRC, car il s'agit d'un outil valide et fiable (Lebel, Simard et al., 2016; Simard et Savard, 2009). Pour obtenir les scores des sous-échelles et le score total de l'IPRC (Simard et Savard, 2009), nous avons additionné les scores des items, cotés en fonction d'une échelle de Likert à cinq points (où le chiffre 0 signifie « pas du tout ou jamais » et le chiffre 4 , « souvent ou tout le temps »). Léquipe de recherche s'est penchée sur les valeurs seuils optimales de l'IPRC pour cibler les survivants dont le degré de PRC mériterait peut-être une intervention professionnelle, degré qu'on qualifie couramment de « cliniquement significatif ». Les scores $\geq 13$ (Simard et Savard, 2015) et $\geq 22$ (Fardell et al., 2018) de la sous-échelle de sévérité ont été proposés, les valeurs seuils ayant été consignées dans le présent article.

\section{CONSTATS}

Les participants $(n=47)$ ont fourni 124 commentaires non sollicités distincts à différents endroits, ici et là dans l'ensemble de la série de sondages, et les commentaires rédigés sur l'IPRC - au nombre de 58 - ont été les plus nombreux, toutes mesures confondues (voir le tableau 1 pour les mesures et le nombre de commentaires pour chacun). C'est au-dessus de l'item 31 («J'appelle mon médecin ou un autre professionnel de la santé ») qu'on a trouvé le plus de commentaires ${ }^{1}(n=6)$. Dans leurs ajouts, les participants précisaient que la section ou l'item d'après ne s'appliquait pas à eux, comme en témoigne cet exemple : "J’y pense rarement [à la possibilité d'une récurrence] et aucune des questions ne s'applique vraiment à moi. »

\section{Dimension qualitative}

La description interprétative a permis de faire ressortir trois grands thèmes ou raisons pouvant expliquer pourquoi les participants ont laissé des commentaires non sollicités dans un sondage sur la PRC : 1) Décrire leur expérience post-traitement; 2) Étoffer ou contextualiser leurs réponses sur la PRC; 3) Exprimer la nécessité d'améliorer les soins oncologiques.

\section{Décrire l'expérience post-traitement}

Les commentaires décrivaient l'incidence du cancer sur la vie des participants, leurs croyances sur la maladie et leurs perspectives sur leur parcours vers la guérison. Ces commentaires laissent croire que les participants ont estimé important

1 Autres endroits où plusieurs commentaires ont été laissés : sous l'item 17 (« Depuis combien de temps songez-vous à la possibilité que le cancer récidive? ») à raison de cinq commentaires; et entre l’item 13 (« Je crois que je suis guéri et que le cancer ne récidivera pas ») et les choix de réponses associés, à raison de quatre commentaires. de raconter leur expérience personnelle, qu'ils jugeaient éclairante pour l'équipe de recherche, bien qu'occultée par le format du sondage. Considérés dans leur ensemble, ces commentaires donnent une idée générale de l'expérience post-traitement des survivants.

Il se dégage des commentaires laissés des opinions polarisées quant à l'incidence du cancer sur la vie des participants. Certains points de vue se traduisaient par des émotions paralysantes ou de la détresse, comme l'illustre ce commentaire : " J'ai de la chance que mon nouveau cancer se soigne très bien, mais je me sens déprimée et personne ne me comprend. "Ces pensées sombres ont entravé la capacité d'agir de certains, notamment la capacité de se fixer des objectifs de vie, a mis à mal leurs relations interpersonnelles ou leur a fait perdre des occasions qu'ils auraient peut-être saisies normalement. Cela dit, certains participants ont souligné des aspects de leur vie qui les préoccupaient plus que le cancer. Par exemple, les personnes s'occupant de jeunes membres de la famille semblaient plus inquiètes à l'idée que le cancer récidive ou qu'elles développent une maladie chronique, indépendante du cancer. Dans une lettre manuscrite jointe au sondage et adressée à l'équipe de recherche, on peut lire : « Le diagnostic de diabète est venu m'ébranler vraiment plus fort [que le cancer], étant donné mes antécédents familiaux... Le diabète me préoccupe, mais je pense à peine au cancer, sauf quand j'ai un rendez-vous de suivi. »

Les participants ont exprimé leurs croyances sur la cause de leur diagnostic ou le risque de récidive. À ce sujet, la génétique et l'âge ont été présentés comme des facteurs de préoccupation impossibles à changer. Selon une participante, « chacun porte en lui des cellules cancéreuses ». Bon nombre de participants ont décrit leurs inquiétudes concernant leur traitement oncologique, considéré comme responsable des effets secondaires ou susceptible d'entraîner une récidive ou un nouveau diagnostic de cancer. Voici un exemple de commentaire : «Une maladie connexe [au traitement] me tracasse. À cause de la chimio, j'ai maintenant une insuffisance cardiaque... Depuis les 16 dernières années, je suis un traitement [cardiaque]. C'est pour veiller à ce que mon cœur fonctionne suffisamment bien pour que je mène une vie normale, malgré quelques limitations. » Par ailleurs, un sous-groupe de participants étaient convaincus que leur cancer était guéri et que les risques de récidive étaient faibles.

Dans leurs témoignages, les participants soulignaient ce que leur avait apporté l'expérience du cancer, notamment un nouveau regard sur la vie. Quelqu'un a écrit : «Le cancer m'a fait réaliser que je ne suis que de passage... Pour moi, c'est essentiel de vivre chaque jour pleinement. La vie est si courte. » D'autres commentaires faisaient état d'une attitude orientée vers l'avenir, empreinte d'une volonté de poursuivre son chemin après le cancer. En bref, cette catégorie regroupe les commentaires où les participants ont raconté leur expérience personnelle, en ajoutant des précisions qui semblaient sortir du cadre du sondage.

Étoffer ou contextualiser les réponses sur la PRC

Les participants ont parfois laissé des commentaires pour contextualiser leurs réponses aux questions sur la peur d'une récidive. Ces ajouts viennent affiner la compréhension des 
facteurs contextuels entourant la PRC, de même que des facteurs temporels qui semblent influencer le vécu des participants à cet égard.

La PRC se décline sur un spectre d'intensité et de fréquence : certains participants s'inquiétaient rarement d'une récidive, d'autres étaient profondément angoissés à cette idée. Parmi les expériences ayant engendré une PRC, mentionnons celles de suivre un traitement hormonal, d'aller à des rendez-vous médicaux et de penser au cancer ou d'en entendre parler. Une participante a écrit : «Une des peurs qui m'habite, une peur incontrôlable, c'est ma mammographie annuelle... Une fois, quand il a fallu refaire ma radio, j'ai perdu connaissance. Je me suis réveillée par terre, couverte d'un drap. " Par ailleurs, les commentaires montraient que les expériences des membres de la famille influencent les pensées et les émotions des participants quant à leur propre crainte du cancer. Par exemple, aux dires d'une participante : "Je ne m'inquiète vraiment pas de la possibilité d'une récidive... En revanche, j'ai peur de développer un cancer de l'ovaire, qui est souvent détecté trop tard, comme ça a été le cas pour ma sœur. »

Les commentaires font également état de multiples sources de PRC et moyens utilisés pour l'apaiser. De nombreux participants ont mentionné le passage du temps, la gratitude ou l'adoption d'attitudes positives, mais ce qui est ressorti le plus souvent est le fait d'avoir une bonne expérience de traitement ou d'appliquer des stratégies d'adaptation (exemples donnés : se réconforter, faire des exercices de relaxation, appeler un professionnel de la santé, se distraire). Un commentaire indique : "Je m'occupe de mon mieux et je fais un peu de tout, sauf penser au cancer. » Les commentaires de cette catégorie viennent contextualiser et préciser la PRC des participants.

Exprimer la nécessité d'améliorer les soins oncologiques

En répondant à un sondage sur la PRC, les participants ont été amenés à réfléchir à leurs interactions positives et négatives avec le système de traitement du cancer. Ajouter des commentaires, cétait une façon pour eux de réclamer des changements dans le système. C'était, semble-t-il, un moyen thérapeutique de raconter leur vécu et de préciser le contexte de leurs réponses. Notons toutefois que la convergence des points de vue sur ce sondage pourrait indiquer une difficulté à mettre les participants en contact avec l'équipe de recherche et les organismes de services pour qu'ils transmettent leurs commentaires.

Certains participants ont remercié l'équipe de recherche de cette étude, à laquelle ils se sont prêtés par désir d'offrir leur contribution. Quelqu'un a écrit : "J'éprouve une immense gratitude pour l'excellence du traitement que j'ai reçu... C'est avec bonheur que j'apporte ma contribution à l'étude, à la mesure de mes moyens. » Ce commentaire dénote une motivation altruiste à participer à la recherche. Une autre participante s'est montrée préoccupée par la situation des patients encore en train d'attendre d'être servis par les prestataires de soins en oncologie: «J'étais déjà dans le système... Je n'ai pas dû attendre plus longtemps pour voir un spécialiste. Mais les autres, eux? » En effet, des participants se sont exprimés sur les obstacles qu'ils ont rencontrés, que ce soit la difficulté à voir un professionnel de la santé ou le manque de coordination des soins post-traitement. Une participante a expliqué comment elle a dû elle-même prendre en charge ses soins : «Je ne crois pas qu’un médecin généraliste puisse prendre en charge un survivant du cancer. J'ai l'impression que c'est à moi de m'en occuper, ce qui m'angoisse un peu. » D'autres ont manifesté une nette préférence pour les soins de suivi assurés par des fournisseurs spécialisés en oncologie, étant d'avis que leur médecin généraliste ne possédait pas les connaissances requises pour bien prodiguer des soins post-traitement. Par ces commentaires, les participants semblent avoir voulu apporter leurs perspectives afin qu'elles contribuent à améliorer les soins aux autres survivants.

\section{Dimension quantitative}

La plupart des personnes ayant laissé des commentaires non sollicités étaient des femmes ( $n=39 ; 83,0 \%$ ) âgées en moyenne de 66 ans (écart-type : + 12,1; étendue : 33-95 ans), titulaires d'un diplôme de premier cycle ou d'un niveau d'études supérieur ( $n=36 ; 76,6 \%$ ) et ayant reçu un diagnostic de cancer du sein ( $n=29 ; 61,7 \%)$ en moyenne 10,7 ans auparavant (écart-type : $+6,8$; étendue : 2 -34). Originaires du Canada pour la plupart ( $n=33 ; 70,2 \%)$, tous les participants habitaient en milieu urbain ( $n=47 ; 100 \%)$. Bien que peu de participants $(n=7,14,9 \%)$ connaissaient un degré de PRC considéré comme cliniquement significatif lorsqu'évalué d'après la valeur seuil de la sous-échelle de sévérité de l'IPRC de $\geq 22$, le degré de PRC était cliniquement significatif pour près de la moitié ( $n=23,48,9 \%$ ) lorsqu'il était évalué d'après la valeur seuil $\geq 13$. Voir les tableaux 3 , 4 et 5 pour connaître tous les détails sur les participants.

Différences entre les participants ayant laissé des commentaires et ceux ne l'ayant pas fait

Après examen de tout l'échantillon des répondants de l'étude principale, il ressort que les personnes ayant laissé des commentaires et celles ne l'ayant pas fait se distinguaient quant à l'âge, le niveau d'éducation et le lieu de naissance. En effet, les personnes ayant laissé des commentaires étaient généralement plus âgées ( $\overline{\mathrm{X}}=66,0$ c. $\overline{\mathrm{X}}=60,9, p<.01$ ), plus nombreuses à détenir un diplôme de premier cycle ou d'un niveau d'études supérieur $(76,6 \%$ c. 54,5 \%, $p$ <.01) et nées au Canada (70,2 \% c. 55,6\%, $p=.04)$. En ce qui concerne le profil clinique, les participants ayant laissé des commentaires souffraient d'un plus grand nombre de comorbidités que les personnes de l'autre groupe $(\overline{\mathrm{X}}=1,26 \mathrm{c} . \overline{\mathrm{X}}=0,79, p=.03)$. Au sein de ce dernier, plus rares étaient les cas de deuxième diagnostic principal, de récidive ou de cancer métastatique $(77,9 \%$ c. $55,3 \%, p<.01)$.

Qui plus est, les scores totaux de l'IPRC étaient inférieurs pour les personnes ayant ajouté des commentaires $(\bar{X}=47,8$ c. $\bar{X}=58,3, p=.01)$. Aucun des scores dichotomisés pour les valeurs cliniquement significatives et non cliniquement significatives ne différait entre les groupes.

\section{DISCUSSION}

Les résultats de la présente étude rendent compte de l'exploration de la nature des commentaires non sollicités laissés dans le cadre d'un sondage sur la PRC. Les commentaires écrits des survivants du cancer semblent être un moyen pour eux de raconter leur réalité post-cancer ou de contextualiser leur vécu par rapport à la PRC. Certains commentaires 
Tableau 5. Peur de la récidive du cancer parmi les groupes

\begin{tabular}{|c|c|c|c|c|c|c|}
\hline \multirow[t]{2}{*}{ Variable } & \multicolumn{2}{|c|}{$\begin{array}{l}\text { Personnes ayant laissé des } \\
\text { commentaires }(n=47)\end{array}$} & \multicolumn{2}{|c|}{$\begin{array}{l}\text { No Narrative Group } \\
\qquad\left(n=955^{\mathrm{a}}\right)\end{array}$} & \multirow[t]{2}{*}{$\begin{array}{l}\text { Test } \\
\text { statistique }\end{array}$} & \multirow[t]{2}{*}{$P$} \\
\hline & $\overline{\mathbf{x}}$ & Écart-type & $\overline{\mathbf{x}}$ & Écart-type & & \\
\hline IPRC - Score total & 47,8 & 25,2 & 58,3 & 28,7 & $t=2,50$ & .01 \\
\hline \multirow[t]{2}{*}{ IPRC - Score de la sous-échelle de sévérité } & 13,2 & 6,5 & 14,7 & 7,7 & $t=1,32$ & .18 \\
\hline & $\mathbf{N}$ & $\%$ & $\mathbf{N}$ & $\%$ & & \\
\hline Niveau de PRC cliniquement significatif $(\geq 13)^{b}$ & & & & & $\chi^{2}=1,58$ & .21 \\
\hline Oui & 23 & 48,9 & 556 & 58,2 & & \\
\hline Non & 24 & 51,1 & 399 & 41,8 & & \\
\hline Niveau de PRC cliniquement significatif $(\geq 22)^{c}$ & & & & & $\chi^{2}=1,03$ & .31 \\
\hline Oui & 7 & 14,9 & 201 & 21,0 & & \\
\hline Non & 40 & 85,1 & 754 & 79,0 & & \\
\hline
\end{tabular}

Note : PRC = peur de la récidive du cancer; IPRC = Inventaire de la peur de la récidive du cancer; $p$ = niveau de signification (test bilatéral); $\mathrm{t}=$ test- $\mathrm{t}$ indépendant; $\chi^{2}$ = test du khi-carré. En raison de données manquantes, il se peut que le total de certaines fréquences n'égale pas le total des colonnes.

a Par manque de données, une personne n'a pas pu calculer les scores de l'IPRC avec la valeur seuil $\geq 13$. La même situation s'est produite pour deux autres personnes, mais avec la valeur seuil $\geq 22$. C'est pourquoi on dénombre 953 et 954 cases pour leurs analyses respectives.

b Valeur seuil de l'IPRC de Simard et Savard (2015). ' Valeur seuil de I'IPRC de Fardell et collaborateurs (2018).

témoignaient d'une volonté de réclamer des changements dans les soins oncologiques. Fait digne de mention, près de la moitié des survivants ayant fourni des commentaires affichait un degré de PRC cliniquement élevé selon la valeur seuil de $\geq 13$ (Simard et Savard, 2015), qui se situe entre les proportions ressorties dans d'autres échantillons hétérogènes de survivants (Lebel et al., 2013; Ng et al., 2019). Autre constat intéressant au sein de l'échantillon : même si le diagnostic remontait à plus de 10 ans en moyenne, le degré de PRC restait cliniquement significatif pour une grande proportion de personnes. Toutefois, les examens systématiques révèlent que la PRC est stable dans le temps (Crist et Grunfeld, 2013; Simard et al., 2013). Cette observation s'expliquerait plutôt par le fait que la plupart des personnes ayant laissé des commentaires étaient des femmes, le sexe féminin étant associé à un degré de PRC supérieur (Galica et al., 2019; Kelada et al., 2019; Ng et al., 2019). Les résultats s'expliqueraient aussi par le constat que le nombre de comorbidités était supérieur chez les personnes ayant laissé des commentaires, celles-ci étant alors peut-être amenées à se rappeler plus souvent leur expérience du cancer. En effet, un nombre accru de symptômes physiques (Hall et al., 2019; Mehnert et al., 2013) et le caractère fréquent des rappels du cancer (Ziner et al., 2012) ont été associés à un degré de PRC supérieur. Notons aussi que les participants savaient que l'objet de l'étude principale était la PRC, l'IPRC ayant été présenté dans un deuxième temps dans la série de sondages à réaliser. Ces facteurs ont peut-être induit un biais de sélection, en ce sens que plus de personnes ayant une PRC élevée ont été amenées à se porter volontaires pour l'étude principale ou à étoffer leurs réponses sur la PRC, en ajoutant alors des précisions sortant des paramètres initiaux de la recherche.

En effet, étant donné la dimension quantitative de l'étude principale, seul un nombre restreint de choix de réponses était proposé aux participants (échelle de Likert). Toutefois, certains d'entre eux ont laissé des commentaires non sollicités sur les sondages (là où il y avait de l'espace, par exemple dans les marges ou entre les questions) ou dans le corps d'une lettre à part, ce qui laisse croire que cétait important pour eux de sortir du cadre de l'étude quantitative pour bien exprimer leur perspective. Cela comporte des implications pour de futures études, comme adopter une approche faisant appel à des méthodes mixtes, l'idée étant de permettre aux survivants qui le souhaitent de raconter leur histoire. À l'avenir, il serait pertinent d'accompagner chaque question quantitative d'une zone de texte libre ou d'entreprendre des projets de recherche qui allient les avantages respectifs des méthodes quantitatives et qualitatives.

Les commentaires non sollicités laissés par les participants témoignent vraisemblablement de leur désir de donner des précisions et du contexte et, par le fait même, de raconter à l'équipe de recherche leur expérience du cancer. Raconter son histoire personnelle a d'ailleurs des bienfaits reconnus (Chelf, Deshler, Hillman et Durazo-Arvizu, 2000; Crogan, Evans et Bendel, 2008). En effet, le récit permet de raconter aux autres des moments charnières de sa vie, un processus qui 
éveille la réflexion et la créativité tout en mettant en relief les aspects émotifs d'un événement (Haigh et Hardy, 2011). Il a été démontré que le récit ou les interventions axées sur la narration atténuent le stress clinique et l'humeur dépressive, et contribuent aussi à la paix d'esprit (Crogan et al., 2008; Wise, Marchand, Roberts et Chih, 2018). Dans un système de santé centré sur les soins physiques, les participants se tournent peut-être vers le récit pour réfléchir à leurs émotions et expériences rattachées au cancer et ainsi mieux les comprendre, ou encore pour faciliter la connexion avec les autres survivants du cancer (Laing, Moules, Sinclair et Estefan, 2019). À cet égard, il se peut que les participants ayant ajouté des commentaires aient simplement ressenti le besoin de mettre en mots les émotions, les obstacles et les victoires qui ont parsemé leur chemin vers la guérison, mais sans forcément chercher à relater leur parcours.

Le récit peut prendre différentes formes (ex. la forme numérique [Lang, Laing, Moules et Estefan, 2019]), mais la littérature retient notre attention sur des types d'écriture bien particuliers, qui semblent les plus pertinents à la lumière des résultats de l'étude. Mentionnons deux types d'écriture ayant été explorés auprès de patients cancéreux : l'écriture expressive, qui invite à s'exprimer librement sur ses expériences personnelles ou des épisodes de la vie (Pennebaker, 1997); et la tenue d'un journal intime, qui permet de verbaliser pensées, idées, sentiments et expériences (Hayman, Wilkes et Jackson, 2012). S'il a certes été démontré que ces formes d'écriture sont bénéfiques pour les patients cancéreux (Williams et Jeanetta, 2016) et leurs proches aidants (Leung et al., 2019), il y a lieu de croire qu'elles profitent davantage aux personnes qui manquent de soutien émotionnel ou viennent juste de recevoir un diagnostic de cancer (Low, Stanton, Bower et Gyllenhammer, 2010). Selon les études, la visée (positive ou négative) de l'activité d'écriture influencerait les retombées psychosociales chez les patients cancéreux (Low et al., 2010; Smith, Anderson-Hanley, Langrock et Compas, 2005).

\section{Limites de l'étude}

Comme elle constitue une analyse de données secondaire, la présente étude comporte potentiellement certaines limites, comme l'impossibilité de clarifier le sens des commentaires laissés et les intentions et objectifs sous-jacents (Thorne, 1998). En outre, les commentaires ajoutés à part étaient laissés à l'interprétation de l'équipe de recherche, selon le contexte et leur emplacement, le contenu de la mesure ou les items autour. Or, les résultats sont à considérer avec prudence et soulignent la nécessité d'une recherche approfondie sur la question.

\section{Implications pour la recherche et la pratique infirmière}

Dans le cadre d'éventuelles études par sondage, les infirmières chercheuses pourraient gagner à inclure des espaces vides pour permettre aux participants d'ajouter des commentaires sur des items qui les rejoignent particulièrement ou d'apporter des clarifications qui seraient autrement laissées de côté. En effet, ce n'est pas la première fois que, dans le cadre d'une recherche par sondage, des commentaires non sollicités sont laissés à même les questionnaires ou dans des lettres jointes (Maliski et Litwin, 2007; Marshall et al., 2019; Warms et al., 2005). Cela dit, à une époque où les sondages en ligne se popularisent, force est de reconnaître qu'il ne sera pas toujours envisageable de fournir de l'espace autour des questions. Limités par la conception de leur plateforme, les sondages virtuels empêcheraient les participants d'étoffer et de contextualiser leurs réponses quand seules sont proposées les options de l'échelle de Likert. Dans un cas du genre, les infirmières chercheuses auraient avantage à intégrer des questions ouvertes facultatives ou des boîtes de texte, où les participants peuvent s'exprimer librement s'ils le souhaitent. Il serait possible d'insérer ces boîtes de texte à divers endroits dans le sondage ou une seule boîte à la toute fin, une fois toutes les questions posées. Ces recommandations ne se limitent pas aux sondages virtuels et pourraient être appliquées à des sondages papier ou aux sondages réalisés sous forme d'entrevues.

Sur le plan de la pratique, les résultats encouragent les infirmières à inciter leurs patients à s'exprimer sur leurs expériences pendant et après leur traitement contre le cancer. Un éventail de moyens s'offrent à eux, selon leurs besoins, par exemple la verbalisation, la tenue d'un journal intime et la production de vidéos numériques. Comme le fait de raconter son histoire atténue le stress et l'anxiété, les infirmières pourraient centrer leur intervention psychosociale sur le récit, en écoutant attentivement l'histoire des patients (Crogan et al., 2008).

\section{Conclusion}

La présente étude, d'une part, apporte des pistes de réponses originales pour expliquer pourquoi certains survivants du cancer laissent des commentaires non sollicités dans le cadre d'un sondage sur la PRC et, d'autre part, indique le type de personnes susceptibles d'être le plus aidées par les interventions axées sur l'écriture expressive pour composer avec la PRC. Les infirmières en oncologie peuvent intégrer des interventions centrées sur l'expression à leurs soins cliniques, et les infirmières chercheuses peuvent inclure aux sondages des espaces pour que le répondant puisse clarifier ses réponses (par des justifications, des explications, du contexte, l'insistance sur un point, etc.).

\section{POINTS À RETENIR}

- On a relevé trois raisons expliquant pourquoi les participants auraient laissé des commentaires non sollicités dans un sondage sur la PRC : 1) Décrire leur expérience post-traitement; 2) Étoffer ou contextualiser leurs réponses sur la PRC; 3) Exprimer la nécessité d'améliorer les soins oncologiques.

- Les survivants du cancer qui semblent les plus susceptibles de laisser des commentaires non sollicités sur les questionnaires auraient une scolarité plus avancées, seraient nées au Canada et auraient obtenu des scores totaux moins élevés à l'IPRC.

- Les infirmières en oncologie peuvent opter pour des interventions centrées sur l'expression pour inciter les survivants du cancer à raconter leur expérience de la maladie.

\section{REMERCIEMENTS}

Les auteurs remercient Weidong Kong pour sa révision des statistiques présentées dans le présent article. 


\section{REFERENCES}

Carlick, A., \& Biley, F. C. (2004). Thoughts on the therapeutic use of narrative in the promotion of coping in cancer care. European Journal of Cancer Care, 13(4), 308-317.

Carver, C. S. (1997). You want to measure coping but your protocol's too long: Consider the Brief COPE. International Journal of Behavioral Medicine, 4(1), 91-100.

Chelf, J. H., Deshler, A. M. B., Hillman, S., \& Durazo-Arvizu, R. (2000). Storytelling: A strategy for living with and coping with cancer. Cancer Nursing, 23(1), 1-5.

Clayton, D. K., Rogers, S. N., \& Stuifbergen, A. (1999). Answers to Unasked Questions: Writing in the Margins. Research in Nursing Q Health, 22, 512-522.

Costa, D. S., Dieng, M., Cust, A. E., Butow, P. N., \& Kasparian, N. A. (2016). Psychometric properties of the Fear of Cancer Recurrence Inventory: An item response theory approach. Psychooncology, 25(7), 832-838. https://doi.org/10.1002/pon.4018

Crist, J. V., \& Grunfeld, E. A. (2013). Factors reported to influence fear of recurrence in cancer patients: A systematic review. Psychooncology, 22(5), 978-986. https://doi.org/10.1002/pon.3114

Crogan, N. L., Evans, B. C., \& Bendel, R. (2008). Storytelling intervention for patients with cancer: Part 2-Pilot testing. Oncol Nurs Forum, 35(2), 265-272. https://doi.org/10.1188/08. ONF.265-272

Eyrenci, A., \& Sertel Berk, H. O. (2018). Validity and reliability of The Turkish Version of Fear of Cancer Recurrence Inventory. Turkish Journal of Oncology. https://doi.org/10.5505/tjo.2018.1759

Fardell, J. E., Jones, G., Smith, A. B., Lebel, S., Thewes, B., Costa, D., . . Butow, P. (2018). Exploring the screening capacity of the Fear of Cancer Recurrence Inventory-Short Form for clinical levels of fear of cancer recurrence. Psychooncology, 27(2), 492-499. https:// doi.org/10.1002/pon.4516

Frid, I., Ohlen, J., \& Bergbom, I. (2000). On the use of narratives in nursing research. Journal of Advanced Nursing, 32(3), 695-703.

Galica, J., Maheu, C., Brennenstuhl, S., Townsley, C., \& Metcalfe, K. (2019). Examining predictors of fear of cancer recurrence using Leventhal's Commonsense Model: Distinct implications for oncology nurses. Cancer Nursing. https://doi.org/10.1097/ NCC. 0000000000000760

Haigh, C., \& Hardy, P. (2011). Tell me a story--a conceptual exploration of storytelling in healthcare education. Nurse Educ Today, 31(4), 408-411. https://doi.org/10.1016/j.nedt.2010.08.001

Hall, D. L., Jimenez, R. B., Perez, G. K., Rabin, J., Quain, K., Yeh, G. Y., . . . Peppercorn, J. (2019). Fear of cancer recurrence: A model examination of physical symptoms, emotional distress, and health behavior change. Journal of Oncology Practice, 15(9), e787-e797. https://doi.org/10. 1200/JOP.18.00787

Hayman, B., Wilkes, L., \& Jackson, D. (2012). Journaling: Identification of challenges and reflection on strategies. Nurse Researcher, 19(3), 27-31.

Hunter, A., \& Brewer, J. D. (2016). Designing multimethod research. In S. N. Hesse-Biber \& R. Burke Johnson (Eds.), The Oxford Handbook of Multimethod and Mixed Methods Research Inquiry. https://doi.org/10.1093/oxfordhb/9780199933624.013.13

Kelada, L., Wakefield, C. E., Heathcote, L. C., Jaaniste, T., Signorelli, C., Fardell, J. E., . . Cohn, R. J. (2019). Perceived cancer-related pain and fatigue, information needs, and fear of cancer recurrence among adult survivors of childhood cancer. Patient Education and Counseling, 102(12), 2270-2278. https://doi.org/10.1016/j. pec.2019.06.022

Laing, C. M., Moules, N. J., Sinclair, S., \& Estefan, A. (2019). Digital storytelling as a psychosocial tool for adult cancer survivors. Oncol Nurs Forum, 46(2), 147.
Lang, M., Laing, C., Moules, N., \& Estefan, A. (2019). Words, camera, music, action: A methodology of digital storytelling in a health care setting. International Journal of Qualitative Methods, 18, 160940691986324. https://doi.org/10.1177/1609406919863241

Lebel, S., Ozakinci, G., Humphris, G., Mutsaers, B., Thewes, B., Prins, J., . . o on behalf of the University of Ottawa Fear of Cancer Recurrence Colloquium attendees. (2016). From normal response to clinical problem: Definition and clinical features of fear of cancer recurrence. Support Care Cancer, 24(8), 3265-3268. https:// doi.org/10.1007/s00520-016-3272-5

Lebel, S., Simard, S., Harris, C., Feldstain, A., Beattie, S., McCallum, M., . . . Devins, G. M. (2016). Empirical validation of the English version of the Fear of Cancer Recurrence Inventory. Qual Life Res, 25(2), 311-321. https://doi.org/10.1007/s11136-015-1088-2

Lebel, S., Tomei, C., Feldstain, A., Beattie, S., \& McCallum, M. (2013). Does fear of cancer recurrence predict cancer survivors' health care use? Support Care Cancer, 21(3), 901-906. https://doi.org/10.1007/ s00520-012-1685-3

Leung, Y. W., Maslej, M. M., Ho, C., Razavi, S., Uy, P., Hosseini, M.-A., . . . Peterkin, A. (2019). Cocreating meaning through expressive writing and reading for cancer caregivers. Journal of Palliative Care, 082585971987153. https://doi.org/10.1177/0825859719871538

Liu, J., Mahendran, R., Chua, S. M., Lam, K. F., Lim, H. A., Kuparasundram, S., . . Griva, K. (2017). Validation of the English and Mandarin versions of the Fear of Cancer Recurrence Inventory in an Asian population. J Health Psychol, 1359105317727819. https:// doi.org/10.1177/1359105317727819

Low, C. A., Stanton, A. L., Bower, J. E., \& Gyllenhammer, L. (2010). A randomized controlled trial of emotionally expressive writing for women with metastatic breast cancer. Health Psychology, 29(4), 460-466. https://doi.org/10.1037/a0020153.supp

Maliski, S. L., \& Litwin, M. S. (2007). Unsolicited written comments: An untapped data source. Oncology Nursing Forum, 34(1), 142-147. https://doi.org/10.1188/07.ONF.142-147

Marshall, C., Linden, W., Ng, A., \& Vodermaier, A. (2019). In the margins: Unsolicited comments of cancer patients across the first year after diagnosis. Canadian Journal of Behavioural Science / Revue canadienne des sciences du comportement, 51(4), 261-268. https://doi.org/10.1037/cbs0000141

Mehnert, A., Koch, U., Sundermann, C., \& Dinkel, A. (2013). Predictors of fear of recurrence in patients one year after cancer rehabilitation: A prospective study. Acta Oncol, 52(6), 1102-1109. https://doi.org/10.3109/0284186X.2013.765063

Moss-Morris, R., Weinman, J., Petrie, K., Horne, R., Cameron, L., \& Buick, D. (2002). The Revised Illness Perception Questionnaire (IPQ-R). Psychology a Health, 17(1), 1-16. https://doi. org/10.1080/08870440290001494

Ng, D. W. L., Kwong, A., Suen, D., Chan, M., Or, A., Ng, S. S., . . Lam, W. W. T. (2019). Fear of cancer recurrence among Chinese cancer survivors: Prevalence and associations with metacognition and neuroticism. Psychooncology, 28(6), 1243-1251. https://doi. org/10.1002/pon.5073

O'Brien, M. R., \& Clark, D. (2010). Use of unsolicited first-person written illness narratives in research: Systematic review. J Adv Nurs, 66(8), 1671-1682. https://doi.org/:10.1111/j.1365-2648.2010.05349.x

Pennebaker, J. W. (1997). Writing about emotional experiences as a therapeutic process. Psychological Science, 8(3), 162-166. https:// doi.org/10.1111/j.1467-9280.1997.tb00403.x

Rammstedt, B., \& John, O. P. (2007). Measuring personality in one minute or less: A 10-item short version of the Big Five Inventory in English and German. Journal of Research in Personality, 41(1), 203212. https://doi.org/10.1016/j.jrp.2006.02.001 
Rosenberg, M. (1965). Society and the adolescent self-image. Princeton, NJ: Princeton University Press.

Scheier, M. F., \& Carver, C. S. (1985). Optimism, coping, and health: assessment and implications of generalized outcome expectancies. Health Psychology 4(3), 219-247.

Simard, S., \& Savard, J. (2009). Fear of Cancer Recurrence Inventory: Development and initial validation of a multidimensional measure of fear of cancer recurrence. Support Care Cancer, 17, 241-251. https://doi.org/10.1007/s00520-008-0444-y

Simard, S., \& Savard, J. (2015). Screening and comorbidity of clinical levels of rear of cancer recurrence. Journal of Cancer Survivorship, 17(418), 1-23. https://doi.org/10.1007/s11764-015-0424-4

Simard, S., Thewes, B., Humphris, G., Dixon, M., Hayden, C., Mireskandari, S., \& Ozakinci, G. (2013). Fear of cancer recurrence in adult cancer survivors: A systematic review of quantitative studies. Journal of Cancer Survivorship, 7, 300-322. https://doi. org/10.1007/s11764-013-0272-z

Smith, S., Anderson-Hanley, C., Langrock, A., \& Compas, B. (2005). The effects of journaling for women with newly diagnosed breast cancer. Psycho-Oncology, 14(12), 1075-1082. The effects of journaling for women with newly diagnosed breast cancer. 10.1002/pon. 912

Thorne, S. (1998). Ethical and representational issues in qualitative secondary analysis. Qualitative Health Research, 8(4), 547-555. The effects of journaling for women with newly diagnosed breast cancer. 10.1177/104973239800800408
Thorne, S. (2013). Interpretive description. In C. T. Beck (Ed.), Routledge International Handbook of Qualitative Nursing Research. Taylor \& Francis.

Thorne, S. (2016). Interpretive description: Qualitative research for applied practice (2nd ed.): Routledge.

Warms, C. A., Marshall, H. M., Hoffman, A. J., \& Tyler, E. J. (2005). There are a few things you did not ask about my pain: Writing on the margins of a survey questionnaire. Rehabilitation Nursing, $30(6)$.

Williams, F., \& Jeanetta, S. C. (2016). Lived experiences of breast cancer survivors after diagnosis, treatment and beyond: Qualitative study. Health Expectations, 19(3), 631-642. The effects of journaling for women with newly diagnosed breast cancer. 10.1111/hex.12372

Wise, M., Marchand, L. R., Roberts, L. J., \& Chih, M.-Y. (2018). Suffering in advanced cancer: A randomized control trial of a narrative intervention. Journal of Palliative Medicine, 21(2), 200207. The effects of journaling for women with newly diagnosed breast cancer. 10.1089/jpm.2017.0007

Ziner, K. W., Sledge, G., Bell, C. J., Johns, S. A., Miller, K. D., \& Champion, V. L. (2012). Predicting fear of breast cancer recurrence and self-efficacy in survivors by age at diagnosis. Oncol Nurs Forum, 39(3), 287-295. 